EGU21-9875, updated on 28 Apr 2021

https://doi.org/10.5194/egusphere-egu21-9875

EGU General Assembly 2021

(c) Author(s) 2021. This work is distributed under

the Creative Commons Attribution 4.0 License.

\title{
Sea ice fragmentation and its role in the evolution of the Arctic sea ice cover.
}

\author{
Adam Bateson ${ }^{1}$, Daniel Feltham², David Schröder ${ }^{2}$, Yanan Wang ${ }^{3}$, Byongjun Hwang ${ }^{3}$, Jeff Ridley ${ }^{4}$, \\ and Yevgeny Aksenov ${ }^{5}$ \\ ${ }^{1}$ Centre for Polar Observation and Modelling, Department of Meteorology, University of Reading, Reading, UK \\ (a.w.bateson@pgr.reading.ac.uk) \\ ${ }^{2}$ Centre for Polar Observation and Modelling, Department of Meteorology, University of Reading, Reading, UK \\ ${ }^{3}$ School of Applied Sciences, University of Huddersfield, Huddersfield, UK \\ ${ }^{4}$ Hadley Centre for Climate Prediction and Research, Met Office, Exeter, UK \\ ${ }^{5}$ National Oceanography Centre Southampton, Southampton, United Kingdom of Great Britain and Northern Ireland
}

The Arctic sea ice cover is not a continuous expanse of ice but is instead composed of individual sea ice floes. These floes can range in size from just a few metres to tens of kilometres. Floe size can influence a variety of processes, including lateral melt rates, momentum transfer within the sea ice-ocean-atmosphere system, surface moisture flux, and sea ice rheology. Sea ice models have traditionally defined floe size using a single parameter, if floe size is explicitly treated at all. There have been several recent efforts to incorporate models of the Floe Size Distribution (FSD) into sea ice models in order to explore both how the shape of the FSD emerges and evolves and its impact on the sea ice cover, including the seasonal retreat. Existing models have generally focused on ocean surface wave-floe interactions and thermodynamic melting and growth processes. However, in-situ observations have indicated the presence of mechanisms other than wave fracture involved in the fragmentation of floes, including brittle failure and melt-induced break up.

In this study we consider two alternative FSD models within the CICE sea ice model: the first assumes the FSD follows a power law with a fixed exponent, with parameterisations of individual processes characterised using a variable FSD tracer; the second uses a prognostic approach, with the shape of the FSD an emergent characteristic of the model rather than imposed. We firstly use case studies to understand how similarities and differences in the impacts of the two FSD models on the sea ice emerge, including the different spatial and temporal variability of these impacts. We also consider whether the inclusion of FSD processes in sea ice models can enhance seasonal predictability. We will also demonstrate the need to include in-plane brittle fracture processes in FSD models and discuss the requirements needed within any parameterisation of the brittle failure mechanism. 